\title{
Um modelo de otimização para priorização em planejamento de Sistemas de Informação
}

\author{
Adiel Teixeira de Almeida Filho, ${ }^{a, *}$, Ana Paula Cabral Seixas da Costa \\ a,*atalmeidafilho@yahoo.com.br, UFPE, Brasil \\ bapcabral@ufpe.br, UFPE, Brasil
}

\begin{abstract}
Resumo
Este trabalho apresenta um modelo para otimizar ganhos competitivos de uma organização advindos da implantação de Sistemas de Informação (SI), atuando em sua priorização. Este artigo aborda parte de um processo mais amplo, o planejamento de $\mathrm{Sl}$, em que serão considerados aspectos estratégicos da organização e outros, relacionados à criticidade de processos que se associam aos Agrupamentos de Informações. A consideração destes aspectos estratégicos, táticos e operacionais será valorada através de uma função de valor aditivo. Neste trabalho é feita alusão a um modelo publicado anteriormente, ao qual serão acrescentadas mudanças na forma como é feita a escolha dos sistemas a serem priorizados, aplicando-se o uso de programação inteira para obter uma ótima solução para o problema de priorização. Ao final do trabalho é apresentado um estudo de caso ilustrando o uso do modelo, em que ele pode ser avaliado.
\end{abstract}

Palavras-chave

Otimização das prioridades em Sistemas de lnformação. Planejamento de Sistemas de lnformação. Apoio multicriterioso à decisão.

\section{Introdução}

0 trabalho apresenta um modelo utilizando métodos de otimização para abordar o problema de priorização de Sistemas de Informação (SI) num contexto mais amplo, que é o planejamento de Sistemas de Informação dentro de uma organização, cuja tarefa de priorização é uma etapa do processo de planejamento. Portanto, este trabalho aborda uma etapa do processo de planejamento e, para isso, utiliza as informações sobre as prioridades da organização obtidas nas etapas anteriores do planejamento de Sl.

Observa-se na literatura a existência de uma linha de pesquisa em torno do planejamento de $\mathrm{Sl}$ e adaptações da metodologia BSP (Business System Planning) (ALMEIDA, 1997, 1999, 2002; ALMEIDA; ALCOFORADO, 1996a, 1996b; ALMEIDA et al., 1991, 1992; ALMEIDA; COSTA, 2002; COSTA et al., 2002, 2003; DUARTE et al., 2005; LOPES; COSTA, 2006, 2007). Este trabalho apresenta um modelo que dá continuidade a essa linha de pesquisa, introduzindo o uso de técnicas de otimização para que o modelo de priorização possa otimizar os ganhos advindos da implantação destes Sls, permitindo considerar restrições sobre aspectos pertinentes a este contexto.

No contexto de planejamento de $\mathrm{Sl}$, em certo momento o gerente precisa tomar decisões sobre quais ferramentas de Tl (Tecnologia da Informação) devem ser escolhidas ou quais projetos devem ser priorizados. A literatura apresenta diversos modelos para apoiar essas decisões, tal como Karsak; Özogul (2009), que mostram um modelo de seleção para a escolha de um ERP (Enterprise Resource Planning), baseando-se nos princípios de QFD (Quality Function Deployment), avaliando as necessidades do usuário sob o ponto de vista deo conceito necessidade do usuário/qualidades de design utilizado no QFD. Outro modelo para escolha de ferramentas de $\mathrm{Tl}$ é proposto por Liang; Li (2008), considerando quatro dimensões, benefício, oportunidades, custos e riscos, utilizando ANP (Analytic Network Process) como método de apoio à decisão.

Cohen (2008) ressalta a necessidade de observar o contexto de planejamento de $\mathrm{Sl}$, destacando 
que em muitas organizações esse planejamento não é explicitado ou é apenas tratado de forma superficial. Cohen (2008) analisou dados de 116 organizações e aponta que a intensidade e o grau de planejamento de SI dependem fortemente da experiência de planejamento da gerência de Sl e do comprometimento da organização com a função $\mathrm{Sl}$, a qual só poderá contribuir para o sucesso do negócio se houver um planejamento eficaz dos recursos aplicados em Sl.

Portanto, o modelo proposto tratará da priorização dos Agrupamentos de Informações, os AGls, que serão implementados ao final do processo de planejamento de SI. O planejamento de SI inclui uma série de etapas, primeiramente fazendo um levantamento da estratégia da empresa, dos processos que fazem parte da organização e das necessidades em termos de SI para automatizar estas atividades. De posse das informações relativas à estratégia da organização, bem como dos aspectos técnicos da implementação (processos englobados pelos AGls) e dos benefícios trazidos pela disponibilidade desses Sls, o modelo proposto neste trabalho permitirá otimizar os ganhos competitivos da organização.

Ao longo do trabalho será apresentada uma visão do processo de planejamento de Sistemas de Informação dentro da metodologia utilizada para o planejamento de Sl, uma adaptação do BSP. Após a contextualização do problema, será mostrado o modelo de priorização de SI proposto, aplicado num estudo de caso.

\section{Contexto do problema}

A metodologia BSP utiliza uma abordagem em que o processo de negócio é a base que estrutura os Sls, considerando que os processos mantêm-se relativamente constantes ao longo do tempo e que os requisitos deles derivados serão refletidos em necessidades de informação não transitórias. Essa metodologia busca transformar a estratégia do negócio em uma estratégia de SI (ZACHMAN, 1982; ALMEIDA; RAMOS, 2002).

Utilizando a visão estratégica do negócio identificam-se os processos do negócio, os dados do negócio e são revisados os sistemas de informações existentes na organização. Em seguida é construída uma arquitetura do Sistema de Informação formalizando as prioridades dos projetos de $\mathrm{Sl}$ para elaborar um plano de ação.

A Figura 1 apresenta um esquema com as fases para o desenvolvimento da metodologia BSP de forma resumida, mostrando as etapas através das

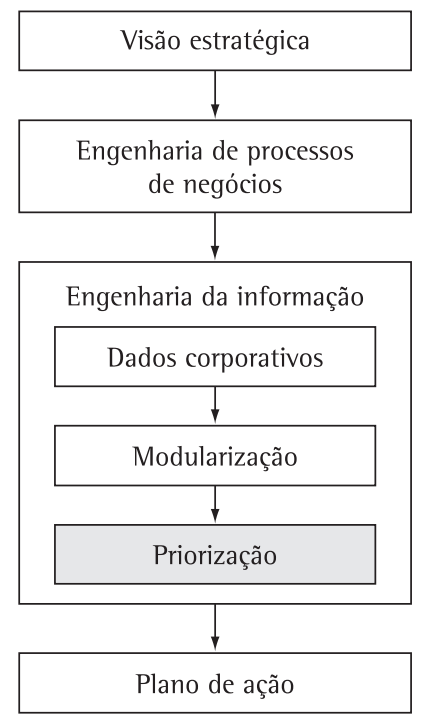

Figura 1. Metodologia para Planejamento de Sistemas de Informação. Fonte: Almeida e Ramos, 2002.

quais será construída uma arquitetura de Sistemas de Informação (ALMEIDA; ALCOFORADO, 1996a).

Ao iniciar o uso da metodologia, é feita uma análise da organização para conhecer a sua estratégia, caso ela não esteja explicitada ao longo do processo de administração. A visão estratégica evidencia-se por meio de fatores estratégicos, visando desenvolver na organização ações orientadas nesse sentido.

Para esses fatores estratégicos, atribue-se peso $\left(e_{i}\right)$, que considera os $n$ fatores estratégicos obtidos anteriormente durante a etapa de visão estratégica. Estes fatores estratégicos são representados pelo vetor da Equação 1, de tal maneira que todos os pesos somem 1 .

$\left\|e_{i}\right\|=\left[\begin{array}{c}e_{1} \\ \ldots \\ e_{n}\end{array}\right]$ onde $0<e_{i} \leq 1$ e $\sum_{i=1}^{n} e_{i}=1$

Após explicitar a missão e a visão estratégica da organização, é desenvolvida a etapa de Engenharia de Processos de Negócio, que consiste em definir todos os processos desenvolvidos ao longo dos vários setores da empresa ou do departamento analisado. De acordo com Davenport (1994), os processos são a estrutura pela qual uma organização faz o que é necessário para produzir valor aos seus clientes.

Já na etapa de Engenharia de Informação, são obtidas informações que levam à construção de uma arquitetura de Sistemas de Informação, que permite abordar questões a respeito de informação partindo 
das classes de dados corporativos e dos processos de negócios da organização. A arquitetura de Sistemas de Informação contempla dentre outros aspectos os Agrupamentos de Informações (AGls). Esta fase da metodologia é composta por três etapas: a de Dados Corporativos, a de Modularização e a de Priorização.

Esse processo inicia-se na fase de Dados Corporativos, onde são definidas as entidades e as classes de dados, ou seja, os grandes grupos de dados corporativos da organização. A partir dessa visão corporativa dos dados, que relaciona um produto a um cliente, começa a etapa de Modularização. Nessa fase obtêm-se os Agrupamentos de Informações (AGls).

Aplica-se um método de aglutinação para que esses AGls contemplem todas as informações, atendendo a um grupo de processos que tenha em comum as mesmas classes de dados envolvidas. $\mathrm{Na}$ etapa da Modularização, se constrói a estrutura informacional da organização, onde os módulos fornecem ferramentas de gestão através de um Agrupamento de Informações que apoia a execução de um conjunto de processos específicos da organização. Finalmente, essa fase encerra com a Priorização, que tem por objetivo fornecer uma posição clara sobre as prioridades das ações de investimento, despesas e necessidades relativas ao desenvolvimento dos AGls.

$\mathrm{Na}$ etapa final do planejamento, é obtido o Plano de Ação, que incluirá o Plano Diretor de Serviço de Informação. 0 Plano Diretor resulta de um encadeamento das informações obtidas nas fases anteriores e consiste numa apresentação das Diretrizes e Metas para o desenvolvimento dos AGls baseada nas prioridades estabelecidas ao longo do processo de planejamento. Por sua vez, essas prioridades se baseiam nas necessidades identificadas pela Engenharia de Processos de Negócio e nos objetivos a serem atingidos, estabelecidos no Plano Estratégico.

Para obter o AGl, as informações são agrupadas pela sua natureza - mais especificamente, tenta-se segmentar as informações relacionadas a processos considerando-se também as classes de dados. Dessa forma, todas as informações que fornecem capacidade de gestão para um determinado(s) processo(s) relacionando-se a determinada(s) classe(s) de dado(s) resultam em Agrupamentos de Informações (AGl).

Portanto, os AGls dependem do contexto a ser analisado, o que quer dizer que vai depender dos processos e especialmente dos dados da organização.

\section{Modelo de otimização proposto para priorização de SI}

A forma como a priorização é feita leva em conta a distribuição de pesos para os diversos aspectos a serem considerados para os AGls. 0 desenvolvimento de um tipo de AGl possui aspectos que favorecem e dificultam sua implementação. Eles serão considerados através dos pesos atribuídos, que representam de certa forma a relação custo/ benefício dessa implementação em várias dimensões. Esta priorização respeitará também as ponderações sob a ótica dos processos a serem atendidos, considerando a forma como esses processos refletirão sua importância relativa na organização sob vários aspectos.

0 modelo proposto neste trabalho é uma adaptação do modelo proposto por Almeida (1999), que se baseava na modelagem de preferências utilizando uma função de valor aditivo (KEENEY; RAIFFA, 1976; VINCKE, 1992), de tal forma que os diversos critérios combinavam-se através de uma função em que era considerada uma situação de incerteza e independência, de preferência, entre os critérios (KEENEY; RAIFFA, 1976). Essa função de valor utilizada no modelo de Almeida (1999) pode ser observada na Equação 2, em que $v_{\mathrm{ij}}$ representa o valor da alternativa $j$ na dimensão do critério $i$, e $w_{\mathrm{i}}$ representa o peso do critério $i$, considerando $k$ critérios. Consideram-se também os valores numa escala normalizada de modo que $0<w_{i} \leq 1$ e que $\sum_{i=1}^{k} w_{i}=1$. É importante salientar que ao utilizar uma função aditiva, os $k$ critérios devem respeitar as exigências para a formação de uma família coerente de critérios (GOMES et al., 2002), respeitando os axiomas da exaustividade, da coesão e da não redundância.

valor da alternativa $j=\sum_{i=1}^{k} v_{i j} w_{i}$

Os critérios a serem utilizados para o sistema de priorização são compostos por dois conjuntos, que apresentam a visão estratégica e a visão por processos. Para isso, é necessário transferir aos AGls a contribuição deles para a visão estratégica e para a visão por processos da organização. Para isso, é feita uma análise durante a etapa de Engenharia de Processos do Negócio, para identificar a participação dos AGls nos processos da organização, e a aderência desses processos contemplados em cada AGl à estratégia da organização. Também são transferidas para os AGls as avaliações relativas ao grau de criticidade de automação e aos aspectos comportamentais das pessoas envolvidas, incorporando-se critérios de processos, concluindo 
assim todos os índices relativos aos Fatores de Ponderação dos AGls.

\subsection{Fatores de ponderação dos AGls}

Os Fatores de Ponderação dos AGls ressaltam as necessidades da organização no processo de priorização. Assim, são considerados $v$ fatores que são agregados de tal forma que fazem uma composição do peso geral de cada AGl. Em meio aos fatores que podem ser agregados, inicialmente serão considerados três fatores como critérios de avaliação para gerar o peso geral de um AGl. Desta forma, serão utilizados os seguintes fatores:

- $i e$ = índice de aderência estratégica;

- $i u=$ índice de comprometimento do usuário; e

- $i c=$ índice de criticidade de automação.

Estes três fatores podem ser detalhados conforme se sugere a seguir:

Índice de Aderência aos Fatores Estratégicos - Este índice tem por objetivo expressar quanto os processos que compõem o AGl participam no desenvolvimento das diretrizes estratégicas, contribuindo para a visão estratégica da empresa.

De início, é obtido o peso estratégico dos processos, e através dele se consegue o índice de aderência dos AGls aos fatores estratégicos. Considere, por exemplo, $p$ processos e $n$ fatores estratégicos, e dessa forma pode-se definir uma matriz, a Equação 3, que estabeleça uma relação entre os fatores estratégicos e os processos da organização, onde os elementos desta matriz representam a participação ou a contribuição de cada processo a cada fator estratégico da organização.

$\left\|r_{i j}\right\|=\left[\begin{array}{cccc}r_{11} & r_{12} & \ldots & r_{1 n} \\ r_{21} & & & \\ \ldots & & & \\ r_{p 1} & \ldots & \ldots & r_{p n}\end{array}\right]$, tal que $0 \leq r_{i j} \leq 1$

$\mathrm{e} \sum_{i=1}^{p} r_{i j}=1, \forall j$

Assim, o peso estratégico dos processos é definido através do vetor pelo vetor da Equação 4, determinado através da relação exposta na Equação 5 , tal que $\sum_{i=1}^{p} p_{i}=1$ e $0 \leq p_{\mathrm{i}} \leq 1$.

$\left\|p_{i}\right\|=\left[\begin{array}{c}p_{1} \\ \cdots \\ p_{p}\end{array}\right]$ $\left\|p_{i}\right\|=\left\|r_{i j}\right\| \cdot\left\|e_{j}\right\|=\left\|\sum_{j=1}^{n} r_{i j} e_{j}\right\|$

Considerando que o número de AGls é igual a $m$, de forma semelhante pode-se obter o índice de aderência dos AGls aos fatores estratégicos, representado pelo vetor abaixo, conforme Equação 6:

$\left\|a e_{i}\right\|=\left[\begin{array}{c}a e_{1} \\ \cdots \\ a e_{m}\end{array}\right]$

Assim como foi feito para a matriz da Equação 3, o grau de relação do AGl com o processo pode ser determinado através da matriz definida da Equação 7:

$\left\|r a_{i j}\right\|=\left[\begin{array}{cccc}r a_{11} & r a_{12} & \ldots & r a_{1 p} \\ r a_{21} & & & \\ \ldots & & & \\ r a_{m 1} & \ldots & \ldots & r a_{m p}\end{array}\right]$, tal que $0 \leq r a_{i j} \leq 1$
e $0 \leq a e_{i} \leq 1, \forall i$

Consequentemente, o índice de aderência dos AGls aos fatores estratégicos pode ser obtido através da relação a seguir, apresentada na Equação 8, considerando $\sum_{i=1}^{m} a e_{i}=1$, e $0 \leq a e_{i} \leq 1$ :

$\left\|a e_{i}\right\|=\left\|r a_{i j}\right\| .\left\|p_{j}\right\|=\left\|\sum_{j=1}^{p} r a_{i j} p_{j}\right\|$

Índice de Comprometimento do Usuário - Este índice tem por objetivo demonstrar a disposição e o interesse do usuário pelo desenvolvimento do AGl e pode ser representado pelo vetor abaixo, conforme Equação 9.

$\left\|a u_{i}\right\|=\left[\begin{array}{c}a u_{1} \\ \ldots \\ a u_{m}\end{array}\right]$, tal que $0 \leq a u_{i} \leq 1$ e $\sum_{i=1}^{m} a u_{i}=1, \forall i$

Este índice pode ser obtido através do nível de comprometimento do usuário com os $p$ processos, representado pelo vetor, representado na Equação 10:

$\left\|u_{i}\right\|=\left[\begin{array}{c}u_{1} \\ \ldots \\ u_{p}\end{array}\right]$, tal que $0 \leq u_{i} \leq 1 \mathrm{e} \sum_{i=1}^{p} u_{i}=1, \forall i$

Desta forma, o índice $a u_{\mathrm{i}}$ é obtido pela relação abaixo (Equação 11):

$\left\|a u_{i}\right\|=\left\|r a_{i j}\right\| .\left\|u_{j}\right\|$ 
Índice de Criticidade de Automação - Este índice tem por objetivo representar o grau de criticidade dos processos envolvidos devido à não automação ou à automação parcial, indicando a necessidade de automação do processo sob o ponto de vista operacional. Assim, este índice agrega dois aspectos: o grau de automação e suas necessidades. Portanto, o Índice de Criticidade de Automação é representado pelo vetor apresentado na Equação 12:

$\left\|a c_{i}\right\|=\left[\begin{array}{c}a c_{1} \\ \ldots \\ a c_{m}\end{array}\right]$, tal que $0 \leq a c_{i} \leq 1$ e $\sum_{i=1}^{m} a c_{i}=1, \forall i$

A obtenção deste índice se dá através do nível de criticidade dos $p$ processos, representado pelo vetor apresentado na Equação 13:

$\left\|c_{i}\right\|=\left[\begin{array}{c}c_{1} \\ \ldots \\ c_{p}\end{array}\right]$, tal que $0 \leq c_{i} \leq 1$ e $\sum_{i=1}^{p} c_{i}=1, \forall i$

Assim, $\left\|a c_{i}\right\|$ é obtido pela Equação 14:

$\left\|a c_{i}\right\|=\left\|r a_{i j}\right\| \cdot\left\|c_{j}\right\|$

\subsection{Peso geral do AGl}

A agregação dos fatores que representam a ponderação dos AGls, através dos índices, permite que se possa obter um índice geral para o AGl. Este índice geral do AGl é representado pelo vetor $\left\|a_{i}\right\|$, conforme Equação 15.

$\left\|a_{i}\right\|=\left[\begin{array}{c}a_{1} \\ \cdots \\ a_{m}\end{array}\right]$

0 vetor da Equação 15 é obtido utilizando a relação ilustrada na Equação 16, que mostra como é feita esta agregação, em que os índices são ponderados em importância de modo que $i e+i c+i u=1$, assim, tal que $\sum_{i=1}^{m} a_{i}=1$, e $0 \leq a_{\mathrm{i}} \leq 1$.

$\left\|a_{i}\right\|=i e\left\|a e_{i}\right\|+i c\left\|a c_{i}\right\|+i u\left\|a u_{i}\right\|$

Almeida (1999) apresenta também uma forma mais geral para os índices operacionais ( $i c, i u)$, na qual estes índices são agregados antes. Assim, poderiam ser considerados nessa etapa apenas dois índices (aderência estratégica e o operacional); alternativa mais abrangente consistiria em utilizar uma forma mais geral para a Equação 16, que será apresentada a seguir.

\subsection{Generalização para obtenção do peso geral do $A G l$}

Em Almeida (1999) encontramos esse procedimento para a generalização do peso geral do AGl, considerando a existência de $v$ fatores de ponderação para os AGls. Dessa forma, o peso dos AGls é obtido considerando o índice relativo dos $v$ fatores de ponderação dos AGls, representado pela Equação 17:

$\left\|i a_{i}\right\|=\left[\begin{array}{c}i a_{1} \\ \ldots \\ i a_{v}\end{array}\right]$, tal que $0 \leq i a_{i} \leq 1$ e $\sum_{i=1}^{v} i a_{i}=1, \forall i$

Assim, o peso dos AGls é obtido pelos fatores representados pela matriz da Equação 18 que configura os $v$ fatores e os AGls.

$\left\|a f_{i j}\right\|=\left[\begin{array}{cccc}a f_{11} & a f_{12} & \ldots & a f_{1 v} \\ a f_{21} & & & \\ \ldots & & & \\ a f_{m 1} & \ldots & \ldots & a f_{m v}\end{array}\right]$, tal que $0 \leq a f_{i j} \leq 1$

e $\sum_{i=1}^{m} a f_{i j}=1, \forall j$

Consequentemente, o peso geral do AGl é dado pela relação mostrada na Equação 19.

$\left\|a_{i}\right\|=\left\|a f_{i j}\right\| .\left\|i a_{j}\right\|=\left\|\sum_{j=1}^{v} a f_{i j} i a_{j}\right\|$

\subsection{Modelo de otimização para priorização de AGls}

A incorporação de um modelo de otimização para a priorização dos AGls permite considerar fatores técnicos, econômicos e aqueles relacionados ao uso dos recursos através da elaboração de restrições a serem respeitadas pelo modelo de otimização.

Uma vez que se possui a função que demonstra os ganhos competitivos advindos da implementação desses Sistemas de Informação a serem priorizados - ou seja, essas questões já foram levantadas e validadas pelos representantes que decidem na organização -, esse tipo de abordagem permite fazer algumas considerações que de outra forma seriam complexas demais para serem percebidas pelos que decidem, por exemplo: uma vez que se possui o ordenamento dos AGls, que serão implementados até que se esgotem os recursos necessários, fica uma pergunta: Será que não seria melhor implantar os $6^{\circ}$ e $9^{\circ}$ colocados no lugar do $4^{\circ}$, por terem custo e necessidades de recursos de implementação similares? 
Obtido o ranking desses AGls em função das prioridades da organização, é claro que esse tipo de raciocínio pode ser feito. Contudo, a maneira como se dá a valoração entre essas duas alternativas $\left(6^{\circ}\right.$ e $9^{\circ}$ colocados juntos, comparados com o $4^{\circ}$ colocado, por exemplo) pode conter algumas incoerências na forma como será feita a análise da sinergia entre as alternativas, onde essas sinergias podem ser positivas ou não, caso haja redundância.

Ao construir um modelo de otimização para priorizar os AGls, está se concordando que a função levantada para contabilizar as vantagens dos AGls para a empresa admite esse tipo de raciocínios e comparações e também que caso haja redundâncias ou qualquer característica particular que impeça a priorização de um conjunto de $n$ alternativas menos prioritárias isoladamente no lugar de uma alternativa que individualmente supera todas as $n$ alternativas, estas (redundâncias/característica particular) podem ser incluídas no modelo através de restrições à função levantada durante o processo de planejamento de $\mathrm{Sl}$ a ser otimizada.

0 modelo de otimização permite também considerar restrições sobre a utilização e/ou capacidade dos recursos disponíveis. Essa seria outra forma de tratar esse problema de priorização de Sl, utilizada por alguns autores, seja investigando o problema em termos da eficiência da priorização (BERNROIDER; STIX, 2006), seja através de uma análise de custo que se baseia na atividade (PEACOCK; TANNIRU, 2005).

0 que se propõe neste trabalho é o uso de um modelo de programação inteira baseado no "problema do saco-mochila", valendo-se do procedimento utilizado por Almeida (1999) para obter a função de valor aditivo que será otimizada e sujeita a restrições que podem dizer respeito tanto à limitação de recursos para a implementação dos projetos de Sistemas de Informação quanto a questões técnicas relativas à redundância ou indisponibilidade de recursos para a implementação simultânea de determinados projetos.

0 "problema do saco-mochila" é um problema de programação linear inteira mista, que permite escolher um subconjunto de alternativas que maximize a função objetivo, satisfazendo um conjunto de restrições. 0 nome "saco-mochila" vem da analogia ao problema em que se deseja escolher um subconjunto de alternativas, onde geralmente se considera que pode-se escolher nenhuma, uma ou mais de uma unidade da alternativa $i$, desejando-se maximizar o valor proporcionado por esses itens (alternativas) que constarão dentro do "saco-mochila", respeitando o limite de peso que se pode carregar nesse "saco-mochila". A modelagem por esse tipo de problema tem uso amplo, sendo bastante utilizado na área financeira para a otimização de carteiras de investimento (WAGNER, 1986). A formulação tradicional do "problema do saco-mochila” está ilustrada na Equação 20, onde $c_{\mathrm{j}}$ são os coeficientes que representam os ganhos obtidos pela escolha da alternativa $j, a_{\mathrm{j}}$ representa no contexto do saco-mochila o peso que a alternativa $j$ ocupará dentro do saco, que em outros contextos pode representar o custo, dada uma limitação de financeira $b$, por exemplo.

$$
\begin{aligned}
& \max \sum_{j=1}^{n} c_{j} x_{j} \\
& \text { s.a } \\
& \sum_{j=1}^{n} a_{j} x_{j} \leq b \\
& x_{j}=0,1 \quad \text { para } j=1,2,3, \ldots n \text {. }
\end{aligned}
$$

No contexto da priorização de Sistemas de Informação, a restrição referente ao problema de otimização pode dizer respeito tanto a uma restrição orçamentária quanto a uma questão técnica da implementação dos Sls priorizados, tal como a capacidade de absorção por setor/usuários, necessidade e disponibilidade de pessoal para o desenvolvimento dos sistemas, entre outras. Dessa forma, a formulação do "problema do saco-mochila" pode ser incorporada ao modelo proposto por Almeida (1999), permitindo comparar um grupo de alternativas com uma alternativa isolada, de forma a ter melhor utilização dos recursos disponíveis, como também incorporar restrições técnicas referentes à implementação desses sistemas.

\section{Aplicação do modelo proposto}

Para a ilustração do modelo proposto, ele será aplicado utilizando-se parte dos dados do estudo de caso apresentado por Almeida (1999), possibilitando a comparação dos modelos de priorização de Sl.

0 trabalho apresentado por Almeida (1999) mostrava um estudo de caso baseado no contexto de uma unidade de negócio de uma empresa de energia elétrica. 0 estudo de caso baseava-se em dados obtidos em cada fase da metodologia adotada de planejamento de SI, onde a ênfase do estudo de caso se dava na fase de priorização. Portanto, serão descritos os passos executados no estudo de caso de Almeida (1999), em que descreve o contexto no qual será aplicado o modelo proposto nesse trabalho para priorização de Sl.

Durante a etapa estratégica, foram obtidos os seguintes fatores estratégicos com seus respectivos pesos: manter nível mínimo de fornecimento de 
serviços $(0,35)$, novos negócios $(0,2)$, aumentar padrão de qualidade $(0,3)$, desenvolvimento de pessoal $(0,15)$. Onde foram levantados os seguintes processos: Infraestrutura, Operações, Serviços, Aquisição, Desenvolvimento Tecnológico, Gerência de Recursos Humanos, Suporte Corporativo e Marketing. E em relação aos AGls, consideraram-se os seguintes agrupamentos: Sistema Físico, Pessoal, Gestão de Serviços, Avaliação de Desempenho de Sistemas e Recursos Financeiros. No início do processo de priorização, foram obtidas informações relativas ao grau de relação entre os processos e os fatores estratégicos estabelecidos. Essas informações são apresentadas na Tabela 1.

Utilizando os valores da Tabela 1 conforme a Equação 5, são obtidos os índices de aderência aos fatores estratégicos para cada processo, resultando nos seguintes indices: Infraestrutura $(0,18)$, Operações $(0,097)$, Serviço $(0,11)$, Aquisição $(0,04)$, Desenvolvimento Tecnológico $(0,205)$, Gerência de Recursos Humanos $(0,145)$, Suporte Corporativo $(0,04)$ e Marketing $(0,18)$.

No que tange aos AGls, estes são relacionados com os processos conforme a Tabela 2 .

Ao aplicar as Equações 8, 11 e 14, são obtidos, respectivamente, os índices de Aderência Estratégica dos AGls, Comprometimento do Usuário e Criticidade de Automação. Os valores destes índices são apresentados na Tabela 3.

Utilizando esses dados será formulado um modelo de otimização que constituirá na ilustração do modelo proposto. Dados os índices da Tabela 3, os pesos a serem usados para valorar a contribuição desses AGls para a organização serão os mesmos considerados por Almeida (1999), $i e=0,45 ; i c=0,2$; $i u=0,35$, que são empregados para compor a função de valor aditivo a ser aplicada. A última linha da Tabela 3 denota o peso global dos AGls, que serão os coeficientes dos AGls na função objetivo a ser otimizada. A forma como esses valores foram obtidos pode ser consultada na Equação 16.

Assim, resta neste momento definir as restrições, ou a restrição a ser considerada no problema de otimização. Para efeito de ilustração do modelo, foi elaborada uma restrição relativa à capacidade da organização de desenvolver projetos de implantação de Sl. Assim será computado o percentual de utilização do pessoal disponivel para o desenvolvimento de cada AGl e, dessa forma, a restrição será que o número de projetos a ser priorizado deve respeitar a capacidade de desenvolvimento de projetos da organização.

Tabela 1. Matriz $\left\|r_{i j}\right\|$. Fonte: Almeida (1999).

\begin{tabular}{ccccc}
\hline & \multicolumn{4}{c}{ Fatores estratégicos } \\
\cline { 2 - 5 } Processos & $\begin{array}{c}\text { Fornecer } \\
\text { serviços }\end{array}$ & $\begin{array}{c}\text { Novos } \\
\text { negócios }\end{array}$ & $\begin{array}{c}\text { Aumentar } \\
\text { qualidade }\end{array}$ & $\begin{array}{c}\text { Desenvolvimento } \\
\text { de pessoal }\end{array}$ \\
\hline Infraestrutura & 0,3 & 0,2 & 0,1 & 0,05 \\
Operações & 0,1 & 0,05 & 0,15 & 0,05 \\
Servicos & 0,05 & 0,05 & 0,25 & 0,05 \\
Aquisição & 0,025 & 0,05 & 0,05 & 0,05 \\
Desenvolvimento tecnológico & 0,2 & 0,3 & 0,1 & 0,3 \\
Gerência RH & 0,1 & 0,1 & 0,15 & 0,05 \\
Suporte Corporativo & 0,025 & 0,05 & 0,05 & 0,15 \\
Marketing & 0,2 & 0,2 & 0,15 & \\
\hline
\end{tabular}

Tabela 2. Matriz $\left\|r a_{i j}\right\|$. Fonte: Almeida (1999).

\begin{tabular}{cllccc}
\hline & \multicolumn{5}{c}{ AGls } \\
\cline { 2 - 5 } Processos & $\begin{array}{c}\text { Sistema } \\
\text { físico }\end{array}$ & Pessoal & $\begin{array}{c}\text { Gestão de } \\
\text { serviços }\end{array}$ & $\begin{array}{c}\text { Avaliação de } \\
\text { desempenho } \\
\text { de sistema }\end{array}$ & $\begin{array}{c}\text { Recursos } \\
\text { financeiros }\end{array}$ \\
\hline Infraestura & 0,3 & 0,05 & 0,2 & 0,15 & 0,3 \\
Operações & 0,25 & 0,15 & 0,3 & 0,25 & 0,05 \\
Serviços & 0,2 & 0,1 & 0,3 & 0,3 & 0,1 \\
Aquisição & 0,4 & 0,1 & 0,1 & 0,1 & 0,3 \\
Desenvolvimento tecnológico & 0,4 & 0,25 & 0,1 & 0,1 & 0,15 \\
Gerência RH & 0,1 & 0,35 & 0,25 & 0,2 & 0,1 \\
Suporte corporativo & 0,1 & 0,3 & 0,1 & 0,1 & 0,4 \\
Marketing & 0,05 & 0,15 & 0,3 & 0,4 & 0,1 \\
\hline
\end{tabular}


Tabela 3. Vetores $\left\|a e_{i}\right\|,\left\|a u_{i}\right\|,\left\|a c_{i}\right\|,\left\|a_{i}\right\|$. Fonte: Almeida (1999).

\begin{tabular}{cccccc}
\hline & \multicolumn{4}{c}{ AGl } & \\
\cline { 2 - 6 } Índices & $\begin{array}{c}\text { Sistema } \\
\text { físico }\end{array}$ & Pessoal & $\begin{array}{c}\text { Gestão de } \\
\text { serviços }\end{array}$ & $\begin{array}{c}\text { Avaliação de } \\
\text { desempenho de } \\
\text { sistema }\end{array}$ & $\begin{array}{c}\text { Recursos } \\
\text { financeiros }\end{array}$ \\
\hline Aderência estratégica & 0,227 & 0,179 & 0,217 & 0,213 & 0,162 \\
Comprometimento do usuário & 0,220 & 0,187 & 0,215 & 0,205 & 0,172 \\
Criticidade automação & 0,215 & 0,175 & 0,215 & 0,205 & 0,189 \\
Peso global do AGl & 0,222 & 0,179 & 0,216 & 0,209 & 0,174 \\
\hline
\end{tabular}

Dessa forma, será considerado que dado um percentual de 100\% do pessoal disponível para a implementação dos projetos, são necessários 20\% do pessoal para o desenvolvimento do AGl referente ao Sistema Físico (SF), 15\% para o Desenvolvimento de Pessoal (DP), 30\% para Gestão dos Serviços (GS), 40\% para Avaliação de Desempenho do Sistema (ADS) e 30\% para Recursos Financeiros (RF).

Finalmente, a formulação do problema de otimização é dada pela Equação 21:

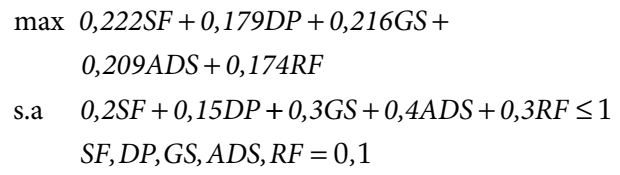

A solução desse problema de otimização pode ser obtida através do Solver do MS Excel, que como resultado para a priorização recomenda a implementação dos seguintes AGls:

- Sistema Físico (SF);

- Desenvolvimento de Pessoal (DP);

- Gestão dos Serviços (GS); e

- Recursos Financeiros (RF).

Apesar do AGl referente à Avaliação de Desempenho do Sistema (ADS) possuir um elevado peso global, foi trocado por outras duas alternativas de menor peso, que juntas permitiram menor desperdício dos recursos disponíveis para a implementação dos projetos (com apenas 5\% de folga ou ociosidade) e resultando em um melhor desempenho na função de valor aditivo, que foi de 0,791 .

Na Tabela 4 pode-se observar a diferença entre os resultados caso a priorização tivesse sido feita sem levar em consideração a possibilidade de maximizar a função de valor aditivo através de um uso mais eficiente dos recursos. Dessa forma, seriam priorizados apenas três AGls, totalizando um valor de 0,647 na função de valor aditivo e resultando numa ociosidade de $10 \%$ dos recursos disponíveis para a implementação dos projetos.
Tabela 4. Resultado utilizando apenas o ranking obtido pela função de valor aditivo até o limite de recursos disponíveis. Fonte: 0 autor.

\begin{tabular}{ccc}
\hline AGl & Uso dos recursos & Peso global do AGl \\
\hline $\begin{array}{c}\text { Sistema físico } \\
\text { Gestão de } \\
\text { serviços }\end{array}$ & 0,2 & 0,222 \\
$\begin{array}{c}\text { Avaliação de } \\
\text { desempenho } \\
\text { de sistema }\end{array}$ & 0,3 & 0,216 \\
Pessoal & 0,4 & 0,209 \\
Recursos & 0,15 & 0,179 \\
financeiros & 0,3 & 0,174 \\
\hline
\end{tabular}

\section{Conclusões}

No artigo foi apresentada uma proposta para que o problema examinado por Almeida (1999) possa ser analisado num contexto em que existem restrições técnicas ou de recursos, possibilitando considerar um conjunto de alternativas com baixo desempenho como uma opção que comporte todas aquelas de baixo desempenho e que agregue o valor de todas aquelas alternativas nos vários critérios considerados dentro do contexto de Planejamento de Sl.

$\mathrm{Na}$ adaptação do modelo de Almeida (1999), foi considerada a mesma função de valor aditivo, modificando apenas a forma como são feitas as comparações entre as alternativas a serem priorizadas.

Para ilustrar a abordagem proposta, foi apresentado um estudo de caso constituído por uma simplificação do estudo de caso mostrado em Almeida (1999), com o objetivo de apenas ilustrar uma situação na qual o modelo adaptado pudesse ser testado.

\section{Referências}

ALMEIDA, A. T. Decision modelling on planning and management of information systems. In: EURO XV/INFORMS XXXIV JOINT INTERNATIONAL MEETING, 1997. Abstracts.. 
ALMEIDA, A. T. Um modelo de decisão para priorização no planejamento de sistemas de informação. Revista Produção, v. 8, n. 2, p. 169-185, 1999.

ALMEIDA, A. T. Multicriteria priorities assignment for information technology based on organisational aspects. International Journal of Operations and Quantitative Management, v. 8, n. 4, p. 251-263, 2002.

ALMEIDA, A. T.; ALCOFORADO, M. M. D. G. Apoio a decisão no planejamento e administração de sistemas de informação. In: ENCONTRO ANUAL DA ASSOCIACC̃OO NACIONAL DOS PROGRAMAS DE PÓS-GRADUAÇÃO EM ADMINISTRAÇÃO, 20, 1996. Anais...

ALMEIDA, A. T.; ALCOFORADO, M. M. D. G. 0 planejamento de sistemas de informação na integração da produção. In: 11 INTERNATIONAL CONGRESS OF INDUSTRIAL ENGINEERING AND XVI NATIONAL CONGRESS OF PRODUCTION ENGINEERING, 1996. Anais...

ALMEIDA, A. T.; BOHORIS, G. A.; STEINBERG, H. Managenment information and decision support system of a telecomunication network. Journal of Decision Systems, v. 1, n. 2-3, p. 213-241, 1992.

ALMEIDA, A. T.; COSTA, A. P. C. S. Modelo de decisão multicritério para priorização de sistemas de informação baseado no método PROMETHEE. Gestão e Produção, v. 9, n. 2, p. 201-214, 2002.

ALMEIDA, A. T.; STEINBERG, H.; BOHORIS, G. A. A decision support system in the management of a telecommunication network. In: SPECIALIZED CONFERENCE ON DECISION SUPPORT SYSTEM, 1, 1991. Proceedings...

ALMEIDA, A. T.; RAMOS, F. S. Gestão da informação na competitividade das organizações. Pernambuco: UFPE, 2002.

BERNROIDER, E. W. N.; STIX, V. Profile distance method: a multi-attribute decision making approach for information system investments. Decision Support Systems, v. 42, n. 2, p. 988-998, 2006.

COHEN, J. F. Contextual determinants and performance implications of information systems strategy planning within South African firms. Information and Management, v. 45, n. 8, p. 547-555, 2008.

COSTA, A. P. C. S.; ALMEIDA, A. T.; GOMES, L. F. A. M. Priorização do portfólio de projetos de sistemas de informação baseado no método TODIM de apoio multicritério a decisão. Revista EPIO, v. 23, n. 1, p. 1-16, 2002.

COSTA, A. P. C. S.; ALMEIDA, A. T.; MIRANDA, C. M. G. Multicriteria support to sort information systems portfolio.
Journal of Academy of Business and Economics, v. 1, n. 2, p. 237-247, 2003.

DAVENPORT, T. H. Reengenharia de processos. como inovar na empresa através da tecnologia da informação. 5 ed. Rio de Janeiro: Campus, 1994.

DUARTE, M. D. O.; GUSMÃO, A. P. H.; ALMEIDA, A. T. Sistema de apoio a decisão com modelo aditivo para priorização de sistemas de informação. Revista Produção Online, v. 5, n. 4, p. 1-11, 2005.

GOMES, L. F. A. M.; GOMES, C. F. S.; ALMEIDA, A. T. Tomada de decisão gerencial: enfoquemulticritério. São Paulo: Atlas, 2002.

KARSAK, E. E.; ÖZOGUL, C. 0. An integrated decision making approach for ERP system selection. Expert Systems with Applications, v. 36, n. 1, p. 660-667, 2009.

KEENEY, R. L.; RAIFFA, H. Decision with multiple objectives preferences and value trade-offs. New York: John Wiley and Sons, 1976.

LIANG, C.; Ll, Q. Enterprise information system project selection with regard to BOCR. International Journal of Project Management, v. 26, n. 8, p. 810-820, 2008.

LOPES, Y. G.; COSTA, A. P. C. S. 0 planejamento e a gestão da tecnologia da informação: um estudo nas empresas pernambucanas. In: ENCONTRO NACIONAL DE ENGENHARIA DE PRODUÇÃO - ENEGEP, 26, 2006. Anais...

LOPES, Y. G.; COSTA, A. P. C. S. Modelo de decisão para seleção de sistemas de informação baseado decisão multicritério e programação inteira 0-1. Revista Gestão Industrial, v. 3, n. 4, p.135-146, 2007.

PEACOCK, E.; TANNIRU, M. Activity based justification of it investments. Information and Management, v. 42, n. 3, p. 415-424, 2005.

VINCKE, P. Multicriteria Decision-Aid. New York: John Wiley and Sons, 1992

WAGNER, H. M. Pesquisa Operacional. 2 ed. São Paulo: Prentice-hall do Brasil, 1986.

ZACHMAN, J. A. Business systems planning and business information control study, a comparison. IBM Systems Journal, v. 21, n. 3, p. 31-53, 1982.

\section{Agradecimentos}

Este trabalho foi parcialmente apoiado pelo CNPq.

\title{
An optimization model to prioritize lnformation Systems planning
}

\begin{abstract}
This work presents a model to optimize Information Systems (IS) organizational payoffs from new investments regarding the process of choosing which projects should be implemented. The paper starts with a wider process, which is IS planning, in which organizational strategic and other technical aspects are considered. To evaluate these aspects, an additive value function taken from another published model is used, which is subsequently adapted to take into account different situations that may occur during the choice process. These adaptations will include the use of integer programming to achieve an optimal solution for this prioritization problem. At the end of the paper, the adapted model will be demonstrated in a case study where it will be possible to observe the different situations that motivated this new model.
\end{abstract}

\section{Keywords}

Information System priorities optimization. Information System planning. Multicriteria decision support. 